\title{
Betti Bases from 4-(3-Pyridazo)-1-naphthol: Synthesis, Coordination Behaviour and Unusual Substitution Reactions
}

\author{
Vanya B. Kurteva, ${ }^{*[a]}$ Lubomir A. Lubenov, ${ }^{[a]}$ Boris L. Shivachev, ${ }^{[b]}$ Rositsa P. Nikolova, ${ }^{[b]}$ and \\ Katharina M. Fromm ${ }^{[c]}$
}

\begin{abstract}
A series of Betti bases from 4-(3-pyridyl)azo-1-naphthol dye, possessing flexible methylene or more constrained arylmethyne spaced $\mathrm{NH}$-containing side-chains, were obtained and characterized. It was shown by NMR spectroscopy that the
\end{abstract}

\section{Introduction}

Coordination compounds are of great importance both in industry and academy. ${ }^{[1]}$ They find applications in medicine ${ }^{[2]}$ and play a fundamental role in synthetic organic chemistry as catalysts for various transformations. ${ }^{[3]}$ Silver(I) complexes in particular are widely studied because of their remarkable medicinal applications. ${ }^{[4]}$ The antibacterial activity of $\mathrm{Ag}(\mathrm{I})$ complexes, well known for many centuries, is among their most attractive biological profiles. Numerous examples of amazingly active complexes with negligible toxicity were reported and summarized in a number of articles. ${ }^{[5]}$ The flexible coordination sphere of silver ions allows the generation of various supramolecular structures with ligands containing nitrogen, oxygen, and/or sulphur donor atoms. ${ }^{[6]}$ Therefore, enormous efforts are devoted to the development of new efficient organic ligands. ${ }^{[7]}$

Azo colorants represent the largest and most important group of synthetic dyes ${ }^{[8]}$ with broad industrial applications, ${ }^{[9]}$ mainly due to their intense colour, chemical stability and low price. Azo-naphthol dyes ${ }^{[10]}$ in particular, are among the most examined dyestuffs due to their optical stability. They play an important role in analytical chemistry as organic complexing reagents ${ }^{[11]}$ due to their capacity to form mostly neutral and hydrophobic complexes with the vast majority of transition

[a] Prof. Dr. V. B. Kurteva, Dr. L. A. Lubenov

Institute of Organic Chemistry with Centre of Phytochemistry Bulgarian Academy of Sciences

Acad. G. Bonchev street, bl. 9, 1113 Sofia, Bulgaria

E-mail: vkurteva@orgchm.bas.bg

[b] Prof. Dr. B. L. Shivachev, Prof. Dr. R. P. Nikolova

Institute of Mineralogy and Crystallography "Acad. Ivan Kostov"

Bulgarian Academy of Sciences

Acad. G. Bonchev street, bl. 107, 1113 Sofia, Bulgaria

[c] Prof. Dr. K. M. Fromm

Department of Chemistry

University of Fribourg

Chemin du Musée 9, CH-1700 Fribourg, Switzerland ligands exist in keto form. The products' structure was confirmed by single crystal XRD of a selected sample. A study on the coordination ability of the ligands with silver(I) was performed, leading to an unexpected substitution reaction.

metal ions. Several members of this class have also displayed a variety of biological activity profiles with medicinal applications. $^{[12]}$

Multicomponent reactions have been approved recently as a powerful tool for waste-free atom efficient synthesis of complex molecules. ${ }^{[13]}$ In particular, the three component Mannich and Betti condensations represent key protocols for the construction of amino alcohols. The Betti reaction, ${ }^{[14]}$ similar to the Mannich reaction, using aldehydes instead of formaldehyde, is typically carried out with 2-naphthol, while the number of examples using 1-naphtol is limited. ${ }^{[15]}$

As a part of our study on the synthesis of new polydentate ligands with potential coordination properties, a series of Betti bases from azo-naphthol dyes were designed. The general concept is to combine both fascinating fragments in a common molecule and to tune the ligands' coordination ability by varying the number and type of heteroatoms in the azo-dye unit and the side-chain. Herein, we present the synthesis of a series of 4-(pyridin-3-yldiazenyl)naphthalen-1-ol ligands possessing a 3-pyridyl Betti type substituent at the 2-position of the naphthyl unit and the examination of their complexation ability towards $\mathrm{Ag}(\mathrm{I})$.

\section{Results and Discussion}

Two types of molecules were designed, possessing a flexible methylene or a more constrained aryl-methyne bridged 3pyridyl unit, in an attempt to analyse the influence of steric hindrance and the type and number of heterocyclic fragments on the complexation behaviour of the ligands. The general formula of the ligands is depicted in Figure 1.

The starting 4-(pyridin-3-yldiazenyl)naphthalen-1-ol $(1)^{[16]}$ was obtained by classical diazo-coupling and was further used without purification. A sample for analyses was purified by flash chromatography on silica gel. The NMR spectra in DMSO- $\mathrm{d}_{6}$ (Figures S1-S6) showed a mixture of two compounds in $83 \%$ and $17 \%$ population of the enol and keto tautomer, respectively. To confirm this assignment, the signals in the spectra 


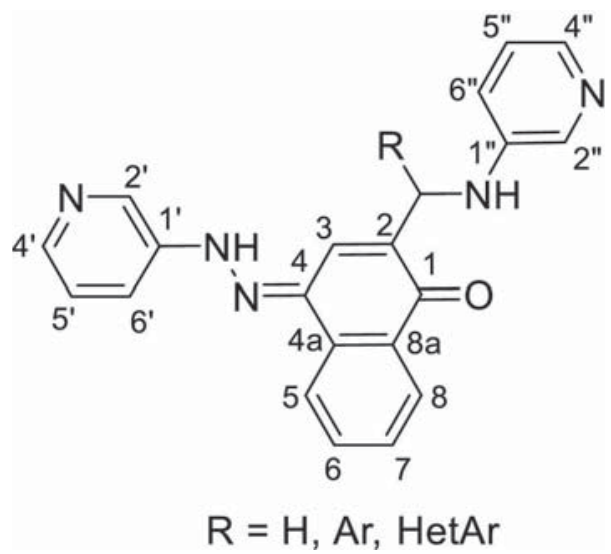

Figure 1. General formula and numbering scheme of the current study objects.

were compared with those of a series of model compounds. The enol and keto forms were fixed as OMe and NMe derivatives by methylation of $\mathbf{1}$. Additionally, compound $\mathbf{2}$, which was expected to exist in the enol form, ${ }^{[17]}$ was obtained by Mannich condensation (Scheme 1). As seen on Figures S19

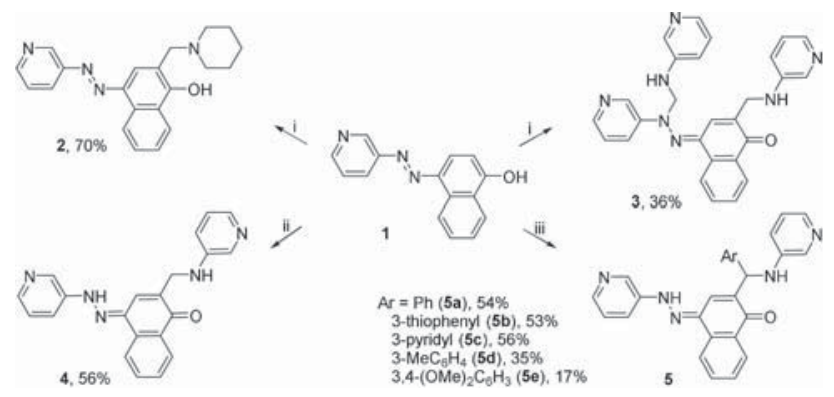

Scheme 1. i) piperidine or 3-aminopyridine, $(\mathrm{HCHO})_{n}, \mathrm{p}$-TSA, benzene, reflux, $3 \mathrm{~h}$; ii) a) 3-aminopyridine, $\left.(\mathrm{HCHO})_{n}, \mathrm{DMSO}^{\circ} 85^{\circ} \mathrm{C}, 1.5 \mathrm{~h}, \mathrm{~b}\right) 1, \mathrm{rt}, 3 \mathrm{~h}$; iii) 3aminopyridine, ArCHO, DMSO, rt, 4-6 days.

and S20, the signals of the major component in the spectra of 1 are fully congruent with those of the model enol compounds 1-OMe and 2.

The starting naphthol 1 was submitted to a Mannich reaction with 3-aminopyridine using standard conditions in refluxing benzene. Surprisingly, the bis-substituted product 3 was isolated instead of the desired ligand 4 (Scheme 1). This problem was solved by performing the reaction in a two-step one-pot protocol with first the formation of the imine at $85^{\circ} \mathrm{C}$ in DMSO, then the condensation reaction at room temperature. The products were characterized by $1 \mathrm{D}$ and 2DNMR spectroscopy and turbo spray mass spectrometry. Compound 4 possesses very limited solubility even in DMSO- $\mathrm{d}_{6}$, and ${ }^{13} \mathrm{C}$ and 2D spectra could not be recorded in a reasonable time scale at room temperature. Unfortunately, the ${ }^{1} \mathrm{H}$ spectra at variable temperature (Figure S34) showed that at $300 \mathrm{~K}$ and $328 \mathrm{~K}$, the pattern is similar, while at $358 \mathrm{~K}$ the product is partially decomposed. The signals in the proton spectrum of 4 were assigned by comparison with those of the better soluble sideproduct 3 (Figures S27-S32). The latter is possible because the spectra of both compounds show identical chemical shifts and patterns, e.g. broad signals for azo-bridged 3-pyridyl and a part of the naphthyl ring protons (Figure 2).

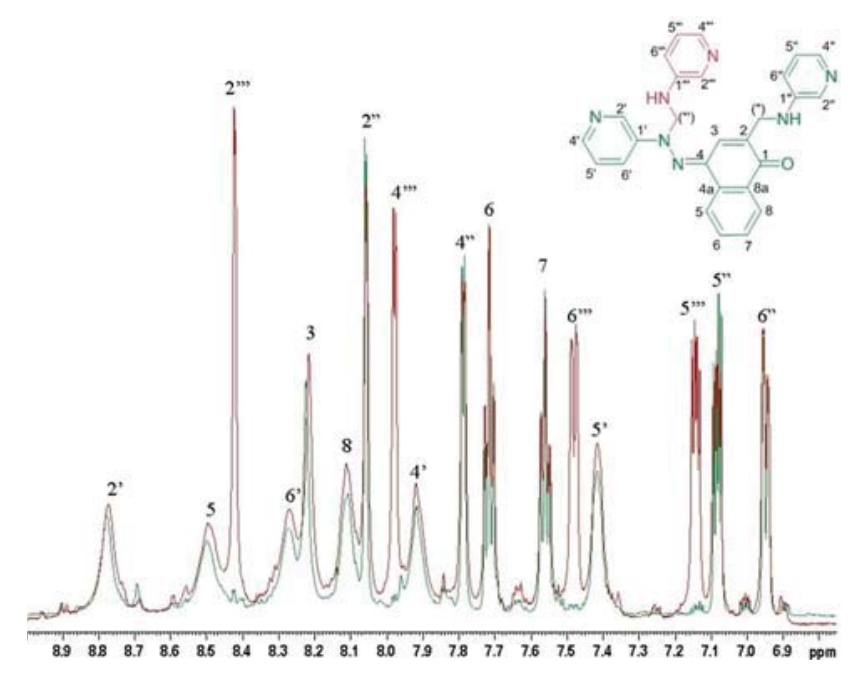

Figure 2. ${ }^{1} \mathrm{HNMR}$ spectra of 3 (brown) and 4 (green) in DMSO- $\mathrm{d}_{6}$ at room temperature.

As compound $\mathbf{3}$ is an example of a pure keto tautomer, and comparting the signals in the spectra of 4 with those of the model compounds, we can assume that the product 4 exists in DMSO solution in the keto form. The structure of the ligand 4 was confirmed by single crystal XRD of the corresponding perchlorate (Figure 3 and Table S1). ${ }^{[18]}$ The free bases however could not be crystallized in our hands from numerous tested solvents and solvent systems.

The aryl-methyne spaced compound group 5 (Scheme 1) was designed with the idea to restrict partially the flexibility of the side-chain. The ligands were obtained by a threecomponent Betti condensation between crude 1, 3-aminopyridine and aromatic aldehyde in good overall yields of up to $56 \%$ in two steps. Ligands $\mathbf{5 a - 5}$ c were synthesised in order to study the influence of an additional heteroatom on the complexation properties, in particular phenyl vs 3-thiophenyl vs 3 -pyridyl, while $\mathbf{5} \mathbf{d}$ and $\mathbf{5 e}$ were designed in an attempt to improve the ligands' solubility. Unfortunately, all compounds possess only limited solubility. The NMR spectra of the products at room temperature showed broad signals for a significant part of the protons due to slow exchange between two sides, which assignment was not reliable. For that reason, 1D and 2D spectra of ligand $5 \mathrm{a}$ were recorded at $343 \mathrm{~K}$. The proton resonances were fully assigned based on the interactions in COSY and NOESY experiments and on the effects in selective NOE spectra at $298 \mathrm{~K}$ (Figures S41-S56). The carbon resonances were partially assigned from HSQC and HMBS spectra (Figur- 


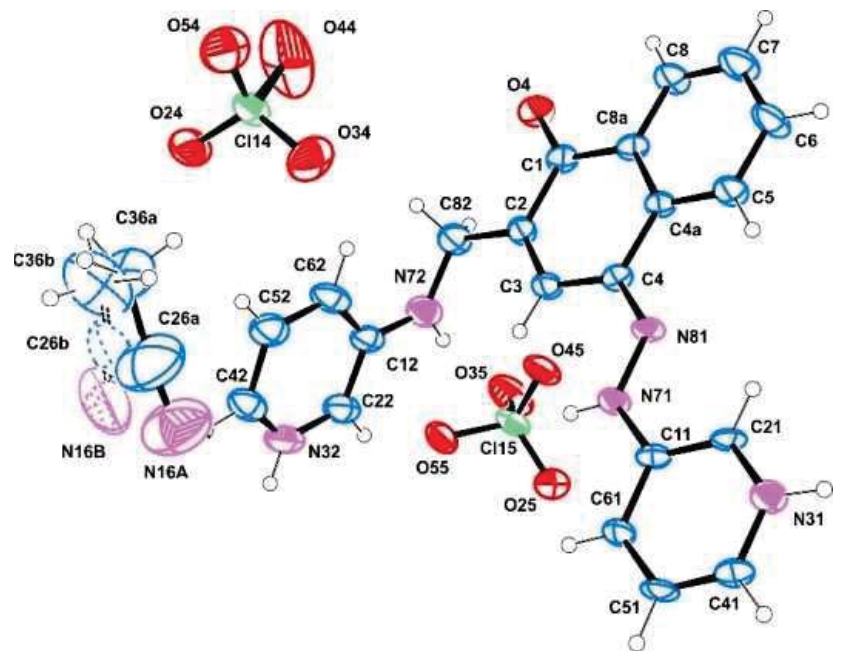

Figure 3. ORTEP view of 4 perchlorate with the atomic numbering scheme; ellipsoids are drawn at 50\% probability, hydrogen atoms are shown as small spheres with arbitrary radii. The solvate acetonitrile molecule is disordered over two positions with major orientation at $70 \%$ (minor component is shown as dashed lines).

es S39 and S40). Some of the quaternary carbon atoms could not be explicitly assigned and are not given.

A comparison between proton resonances of $5 \mathrm{a}$ and its methylene bridged analogue 4 (Figure 4) showed that only $\mathrm{CH}$ -

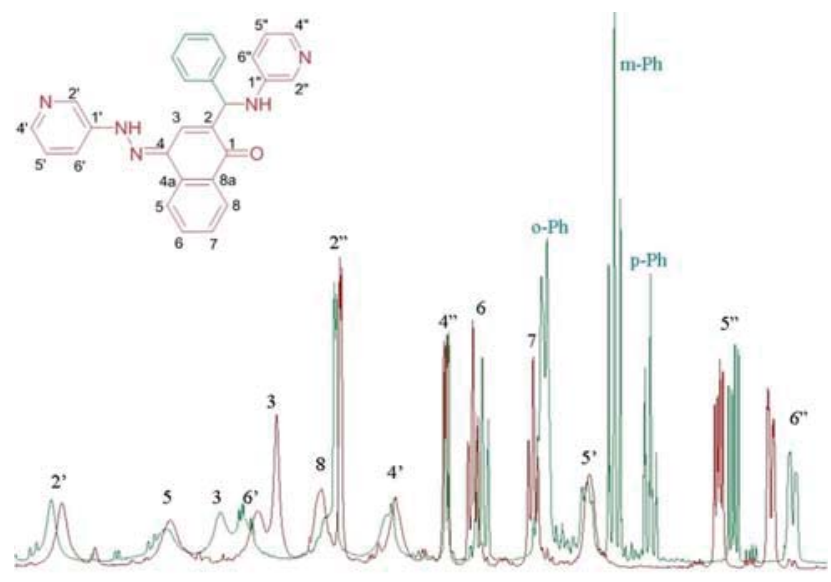

$\begin{array}{lllllllllllllllllllllllllll}8.8 & 0.7 & 8.6 & 0.5 & 8.4 & 0.3 & 0.2 & 8.1 & 8.0 & 7.9 & 7.6 & 7.7 & 7.6 & 7.5 & 7.4 & 7.3 & 7.2 & 7.1 & 7.0 & \text { PF }\end{array}$

Figure 4. ${ }^{1} \mathrm{H}$ spectra of 3 (brown) and 5 a (green) in $\mathrm{DMSO}^{-\mathrm{d}_{6}}$ at room temperature.

6" and $\mathrm{CH}-3$ signals are slightly shifted downfield and upfield, respectively, and are more broadened in the Betti base with respect to methylene bridged compound.

The structures of the ligands $5 \mathrm{~b}-5 \mathrm{e}$ were assigned by comparison of the NMR spectra with those of $5 \mathrm{a}$. As illustrated on Fig. 5 on the example of $\mathbf{5} \mathbf{b}$, the signals for the skeleton protons have identical chemical shifts as those of $5 \mathrm{a}$. This pattern is valid for all ligands $\mathbf{5}$.

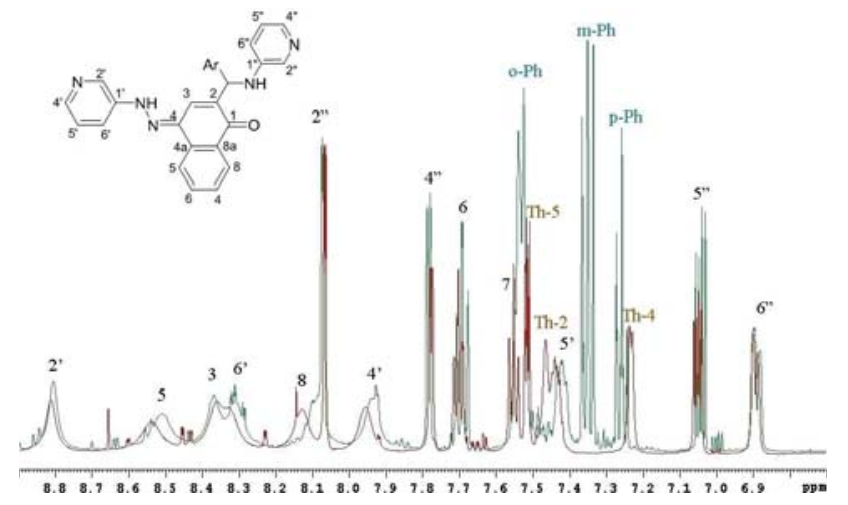

Figure $5 .{ }^{1} \mathrm{H}$ spectra of $\mathbf{5} \mathbf{a}$ (green) and $\mathbf{5} \mathbf{b}$ (brown) in DMSO- $\mathrm{d}_{6}$ at room temperature.

The coordination ability of compounds 5 towards $\mathrm{Ag}(\mathrm{I})$ was studied using different anions, solvents, and dilutions, while maintaining a ratio of 1:1 between the ligand and the metal ion. In all cases a black solid phase was formed upon mixing, either immediately or after several hours or days depending mainly on the dilution. The amount of solid always exceeded the expected mass of $\mathrm{Ag}(0)$, which led to the assumption that coordination took place. Unfortunately, the residues were completely insoluble in any solvent or solvent system to record NMR spectra and/or to be recrystallized.

Only in one case did we obtain a crystal appropriate for XRD analyses using slow evaporation of an extremely diluted ethanol solution $\left(10^{-3} \mathrm{M}, 0.05 \mathrm{mmol}\right.$ in $\left.50 \mathrm{ml}\right)$ of ligand $5 \mathrm{~b}$ and silver(I) nitrate. Surprisingly, the analysis showed that instead of desired silver complex, compound 6 was formed in which the side-chain pyridyl group was unexpectedly replaced by an ethyl group (Scheme 2, Figure 6, and Table S1). ${ }^{[18]}$
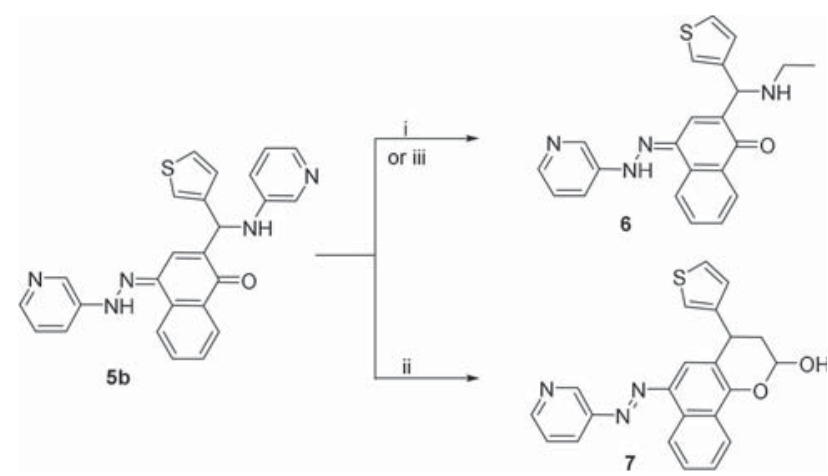

Scheme 2. i) $\mathrm{AgNO}_{3}$, $\mathrm{EtOH}$, rt; ii) $\mathrm{CH}_{3} \mathrm{CHO}, \mathrm{DMF}$, rt; iii) $\mathrm{CH}_{3} \mathrm{CHO}$, EtOH, rt.

The observed transformation could be explained by a reaction between $\mathbf{5} \mathbf{b}$ and acetaldehyde, obtained by oxidation of a part of ethanol, going most probably via an iminium state. To confirm this suggestion, the same ligand was submitted to a reaction with acetaldehyde in DMF, which was used as a solvent in order to reach a sufficient concentration of $3 \times 10^{-2} \mathrm{M}$ 


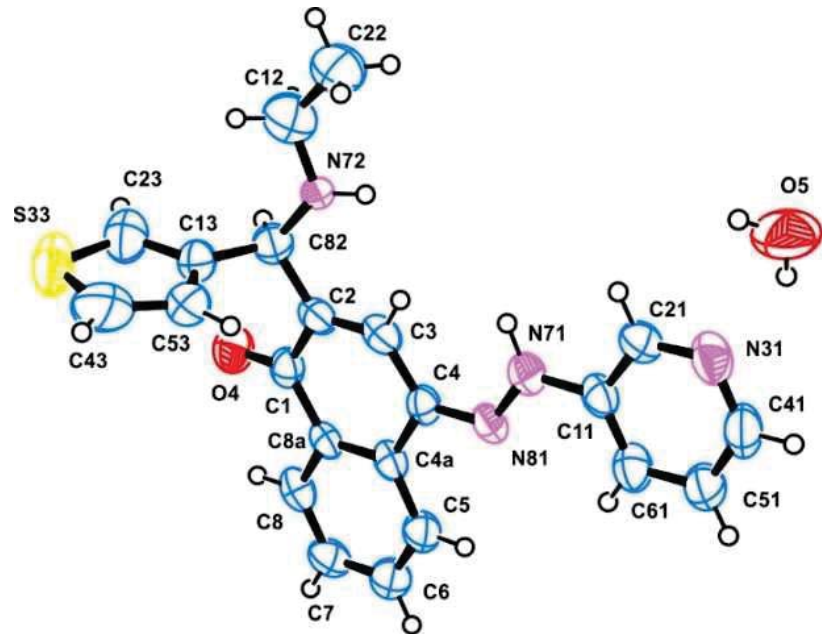

Figure 6. ORTEP view of 6 with the atomic numbering scheme; ADP are drawn at $50 \%$ probability, hydrogen atoms are shown as small spheres with arbitrary radii. The thiophene ring sulphur (S33) and C43 atom are disordered over two positions with major orientation at 53\% (minor component not shown on the drawing).

for the reaction. The isolated product was however identified by NMR and XRD as compound 7 (Scheme 2, Figures S65-S69, Figure 7, and Table S1). ${ }^{[18]}$ In order to avoid this, the experiment

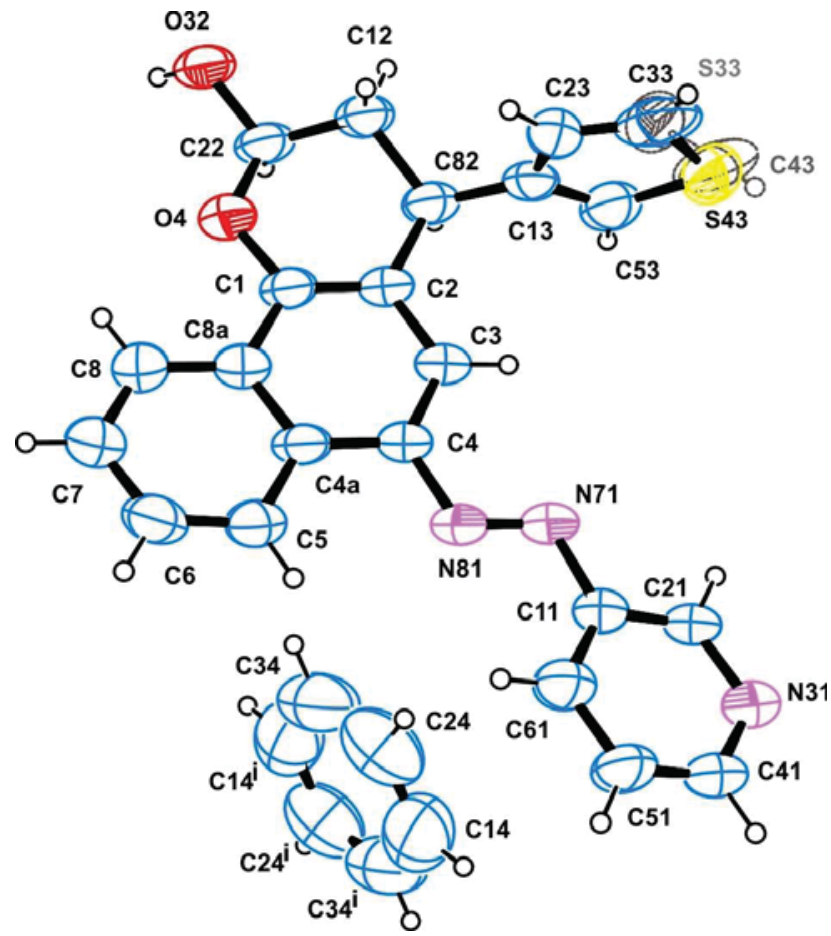

Figure 7. ORTEP view of 7 with the atomic numbering scheme; ADP are drawn at $40 \%$ probability, hydrogen atoms are shown as small spheres with arbitrary radii. The thiophene ring sulphur (S33) and C43 atoms are disordered over two positions with major orientation at 73\% (minor component is shown as dashed lines). was then performed in ethanol suspension. Among a series of ethanol-acetaldehyde condensation products, which were isolated by chromatography, compound 6 could be unambiguously attributed in the NMR spectrum of the reaction mixture, confirming thus the proposed pathway (Fig. S70-74).

\section{Conclusions}

A series of Betti bases from 4-pyridylazo-1-naphthol were efficiently synthesised and characterized in solution and in the solid state. The compounds can be divided into two groups, e.g. the ones possessing i) a flexible methylene or ii) a more constrained arylmethyne bridged 3-pyridyl side-chain. It was demonstrated that the ligands exist in keto tautomeric form independently of the type of the spacer group. The study on the complexation ability of the ligands was unsuccessful due to the lack of solubility of the formed products. An unexpected result with side-chain pyridyl group replacement was obtained and the product was analysed by XRD. A possible pathway was suggested and synthetically confirmed.

\section{Supporting Information Summary}

Supporting Information includes experimental details, characterization and crystallography data of all synthesised compounds and Appendix, containing original NMR and UV spectra.

\section{Acknowledgements}

The financial support by Swiss National Science Found, project 160515"SupraMedChem@Balkans.eu" under SCOPES 2015-2018, by The Bulgarian Science Fund, project DCOST-01-23 and infrastructure projects UNA-17/2005, DRNF-02-13/2009 and DRNF-02/ 01 , and by The EU, COST Action CA15106 "C-H Activation in Organic Synthesis" (CHAOS), is gratefully acknowledged.

\section{Conflict of Interest}

The authors declare no conflict of interest.

Keywords: 4-(3-pyridylazo)-1-naphthol · Betti condensation · 3aminopyridine $\cdot \mathrm{NMR} \cdot \mathrm{XRD}$

[1] a) S. Jurisson, D. Berning, W. Jia, D. Ma, Chem. Rev. 1993, 93, 1137-1156; b) K. L. Haas, K. J. Franz, Chem. Rev. 2009, 109, 4921-4960; c) H. Willms, W. Frank, C. Ganter, Organometallics 2009, 28, 3049-3058; d) H.C. Aspinall, Chem. Rev. 2002, 102, 1807-1850.

[2] a) C. W. Schwietert, J. P. McCue, Coord. Chem. Rev. 1999, 184, 67-89; b) J. Reedijk, Macromolec. Symp. 2008, 270, 193-201.

[3] a) P. B. Weisz, Annual Rev. Phys. Chem. 1970, 21, 175-196; b) J. Halpern, Annual Rev. Phys. Chem. 1965, 16, 103-124.

[4] a) E. M. Hetrick, M. H. Schoenfisch, Chem. Soc. Rev. 2006, 35, 780-789; b) A. Kascatan-Nebioglu, M. J. Panzner, C. A. Tessier, C. L. Cannon, W. J. Youngs, Coord. Chem. Rev. 2007, 251, 884-895; c) S. I. M. Rafique, A. Nasim, H. Akbar, A. Athar, Biotechnol. Mol. Biol. Rev. 2010, 5, 38-45; d) K. Fromm, Nature Chem. 2011, 3, 178; e) S. Eckhardt, P. S. Brunetto, J. Gagnon, M. Priebe, B. Giese, K. M. Fromm, Chem. Rev. 2013, 113, 47084754 . 
[5] a) S. Djokić, Bioinorg. Chem. Appl. 2008, Article ID 436458, 7 pp.; b) K. Vasilev, J. Cook, H. J. Griesser, Expert Rev. Med. Dev. 2009, 6, 553-567; c) O. Gordon, T. Vig Slenters, P.S. Brunetto, A. E. Villaruz, D. E. Sturdevant, M. Otto, R. Landmann, K. M. Fromm, Antimicrob. Agents Chemother. 2010, 54, 4208-4218; d) N. Loginova, A. Chernyavskaya, G. Polozov, N. Osipovich, T. Koval'chuk, A. Gres, Y. Halauko, A. Halauko, R. Zheldakova, Y. Faletrov, I. Azarko, Mini-Rev. Org. Chem. 2013, 10, $227-$ 240; e) P. Pallavicini, G. Dacarro, Y. A. Diaz-Fernandez, A. Taglietti, Coord. Chem. Rev. 2014, 275, 37-53.

[6] H. W. Roesky, M. Andruh, Coord. Chem. Rev. 2003, 236, 91-119.

[7] C. Copéret, A. Comas-Vives, M. P. Conley, D. P. Estes, A. Fedorov, V. Mougel, H. Nagae, F. Núñez-Zarur, P. A. Zhizhko, Chem. Rev. 2016, 116, 323-421.

[8] a) K. Venkataraman, The chemistry of synthetic dyes, Academic Press, New-York and London, 1970; b) H. Zollinger, Color Chemistry, Synthesis, Propertiesand Application of Organic Dyes and Pigments, Wiley-VCH, Weinheim, 2003; c) R. M. Christie, Azo dyes and pigments, RSC Publishing, 2001.

[9] a) P. Gregory, High-Technology Applications of Organic Colorants, Plenum Press, New York, 1991; b) Industrial Dyes. Chemistry, Properties, Applications, Hunger, K. (Ed.), Wiley-VCH Verlag GmbH \& Co. KGaA, 2003.

[10] A. D. Towns, Dyes Pigm. 1999, 42, 3-28.

[11] a) T. M. Florence, Y. Farrar, Anal. Chem. 1963, 35, 1613-1616; b) K. L. Cheng, R. H. Bray, Anal. Chem. 1955, 27, 782-785; c) R. G. Anderson, G. Nickless, Analyst 1967, 92, 207-238; d) K. Pytlakowska, V. Kozik, M. Dabioch, Talanta 2013, 110, 202-228.

[12] a) C. Huggins, J. Pataki, PNAS 1965, 53, 791-796; b) E. Węglarz-Tomczak, Ł. Górecki, Chemik 2012, 66, 1298-1307.
[13] a) T. J. J. Müller, Multicomponent reactions, McGraw-Hill Education, 2016; b) I. Ugi, A. Dömling, W. Hörl, Endeavour 1994, 18, 115-122; c) C. de Graaff, E. Ruijter, R. V. A. Orru, Chem. Soc. Rev. 2012, 41, 3969-4009; d) A. Dömling, W. Wang, K. Wang, Chem. Rev. 2012, 112, 3083-3135.

[14] a) Z. Wang, Comprehensive Organic Name Reactions and Reagents, John Wiley \& Sons, Inc., 2010; b) C. Cardellicchio, M. A. M. Capozzi, F. Naso, Tetrahedron: Asymm. 2010, 21, 507-517.

[15] I. Szatmári, F. Fülöp, Tetrahedron 2013, 69, 1255-1278.

[16] S. H. Etaiw, Y. M. Issa, Kolorisztikai Ertesito 1978, 20, 37-41.

[17] a) L. Antonov, V. Deneva, S. Simeonov, V. Kurteva, D. Nedeltcheva, J. Wirz, Angew. Chem. Int. Ed. 2009, 48, 7875-7878; b) L. M. Antonov, V. B. Kurteva, S. P. Simeonov, V. V. Deneva, A. Crochet, K. Fromm, Tetrahedron 2010, 66, 4292-4297; c) L. Antonov, V. Deneva, V. Kurteva, D. Nedeltcheva, A. Crochet, K. M. Fromm, RSC Adv. 2013, 3, 25410-25416; d) V. B. Kurteva, L. M. Antonov, D. V. Nedeltcheva, A. Crochet, K. M. Fromm, R. P. Nikolova, B. L. Shivachev, M. S. Nikiforova, Dyes Pigm. 2012, 92, 1266 1277.

[18] Crystallographic data (with structure factors) for the structural analysis have been deposited with the Cambridge Crystallographic Data Centre, Nos. CCDC - 1854785 (4 perchlorate), 1873276 (6) and 1873277 (7) Copies of this information may be obtained free of charge from: The Director, CCDC, 12 Union Road, Cambridge, CB2 1EZ, UK. Fax: +44(1223)336-033, e-mail:deposit@ccdc.cam.ac.uk, or www: www.ccdc.cam.ac.uk. 